\title{
Indicador Innovación y Cobertura Solar
}

\author{
Erwin Medina Josefa ${ }^{\text {al }}$ \\ ${ }^{a}$ El Colegio del Estado de Hidalgo. Calle Miguel Hidalgo No. 618, colonia Centro, Pachuca de Soto, Hidalgo. C.P. 42000.
}

\section{Resumen}

En este texto se reconoce la importancia de los indicadores urbanos sustentables, así como del amplio y muy recorrido contexto, que a través de los años ha tenido el desarrollo sustentable, que junto con el cambio climático son los requirentes y, al mismo tiempo, promotores de indicadores que contribuyan al mejoramiento de la calidad de vida de las personas que habitan en las ciudades. La energía es fundamental para el desarrollo de las sociedades; el objetivo de desarrollo sostenible número siete es el interés oficial de la ONU en esta materia, el marco es la agenda 2030. Del conjunto de energías renovables, es la opción solar en conjunto con el aditamento tecnológico panel solar, la opción que cuenta con el mayor potencial para su implementación en el ámbito doméstico. Entonces, de acuerdo al panorama descrito, ha sido tarea desarrollar un indicador exploratorio para conocer la cobertura de la energía solar en los hogares mexicanos por estado de la República, utilizando como primer insumo hogares con energía eléctrica por panel solar. Se retoma la propuesta de enfoque "eficiencia energética desde el contexto de las "buenas prácticas' y las energías limpias". Bajo el enfoque mencionado se agruparon los datos relativos, se normalizaron y se ponderaron. Se realiza una revisión nacional para el año 2014 utilizando datos de INEGI. Se obtiene entonces un ranking, Durango y Sonora son los primeros de la lista. Se proporcionan algunas reflexiones respecto del ejercicio y de las áreas de oportunidad existentes.

Palabras Clave: energía solar, panel solar, hogares con panel solar, indicador urbano.

\section{Introducción}

Primera premisa: la permanente búsqueda de un modelotipo de ciudad sustentable inspirado por el constructo teóricoanalítico desarrollo sustentable de la ONU, conduce a que en el presente trabajo se describa el proceso de elaboración de un indicador urbano sustentable.

Segunda premisa: es de interés en este texto el tema particular de la energía a través de las renovables, por ello se toma como elemento principal la tecnología panel solar y su aplicativo en los hogares mexicanos, siendo consonantes con la realidad actual en por lo menos tres aspectos: (1) la producción de energía eléctrica es uno de los tres procesos que genera mayor cantidad de Gases de Efecto Invernadero (GEI) en México; (2) de acuerdo a Hábitat III el crecimiento de la población se evidenciará con la concentración de esta mayormente en las ciudades; y (3) el periodo de transición energética en sí mismo es un proceso en el que las naciones del mundo se ven obligadas a desarrollar y consolidar una industria respecto de las energías limpias, de tal suerte que la electricidad que consumen hogares e industria sea producida por una variedad de energías limpias que posibilite una matriz energética sostenible y equilibrada.

Entonces, se retoma el tema de los indicadores urbanos sustentables, línea de investigación que hoy contribuye de forma importante al diseño de políticas públicas que bajo el contexto del desarrollo sustentable (en una lógica de optimismo tecnológico) contribuye a la calidad de vida de las personas. Los indicadores son monitores de una realidad.

Autores como Rollo Home (2014) señalan que el cambio climático se enfoca cada vez más en la resiliencia energética, y la preocupación en el caso de las ciudades radica en que la presión sobre las infraestructuras es cada vez más fuerte.

$\mathrm{Se}$ prioriza un interés ambiental. Una forma de contrarrestar los GEI es precisamente mediante el bajo aprovechamiento de la energía solar que se registra en México. Así, por un lado, se trata de disminuir los GEI, pero por otro lado se trata de promover una discusión de un tipo-modelo ciudad sustentable, partiendo de los planteamientos propios del discurso oficialista del desarrollo sustentable.

De forma particular, para desarrollar el presente trabajo las categorías de análisis son: (1) los hogares mexicanos con energía eléctrica por panel solar; y (2) el enfoque de la eficiencia energética desde el contexto de las "buenas prácticas" y las energías limpias.

Así, se creó el Indicador Innovación y Cobertura Solar (IICS) que permite generar un ranking nacional en materia de cobertura de energía solar en los hogares mexicanos. Un ambiente propicio para la energía solar es aquel en el que el desarrollo tecnológico se ha detonado, aquel en el que se ha incentivado la implementación a través de política pública, y aquel en el que se han socializado los beneficios.

\section{Fundamentos}

\subsection{Indicador urbano sustentable}

En el marco del desarrollo sustentable, del monitoreo respecto de sus avances, así como en la necesidad de sus ajustes en sus diferentes ámbitos de acción, es que los indicadores se desarrollan, se aplican, se monitorean, se

\footnotetext{
${ }^{1}$ Autor en correspondencia.

Correos electrónicos: medina.erwin@gmail.com (Erwin Medina Josefa)
} 
evalúan y se retroalimentan. El desarrollo sustentable es la agenda que, de acuerdo con el cambio climático (como problema global reconocido) delinea políticas de acción en cada país, políticas (policy) que se ajustan de acuerdo a las posibilidades técnicas, tecnológicas, operativas, económicas y políticas (politics).

En este sentido, los indicadores tienen su trayectoria en el ámbito de la sustentabilidad; estos se remontan a los años 70's. El informe Brundtland en 1987 fue un parteaguas en este sentido al integrar tres ámbitos que perduran hasta hoy día, el social, el ambiental y el económico, amén del institucional.

Gutiérrez Garza (2012:13) conceptualiza desarrollo sustentable como: "[...] el cuidado del medio ambiente, así como la integridad de los ecosistemas, las relaciones sociales solidarias orientadas hacia la equidad y los entornos institucionales de la política para el desarrollo de la gobernanza democrática", lo anterior como la autora misma lo enmarca, en reconocimiento de un modelo de crecimiento.

Algunos autores establecen, independientemente del tema de la sustentabilidad, las propiedades estratégicas de un fenómeno para concretar un indicador; tal es el caso de autores como Lazarsfeld (1973) que se remiten hasta la parte de abstraer la realidad de un fenómeno observado. Altamirano (1992) por su lado refiere el tema de la operacionalización de los conceptos.

En el terreno de la política pública, Mondragón (2002:52) refiere que no hay una definición oficial de indicador. La autora destaca una aproximación “«[...] son medidas verificables de cambio o resultado [...]»". Raymond A. Bauer (autor citado por Mondragón 2006:52) destaca que "los indicadores sociales [...] son estadísticas, serie estadística o cualquier forma de indicación que nos facilita estudiar donde estamos y hacia donde nos dirigimos con respecto a determinados objetivos y metas [...]". Siguiendo esta cita de Bauer de 1966, el autor también refiere que el indicador permitirá la evaluación de programas específicos a través de la medición precisamente de los impactos; dicho esto, entonces los indicadores son una herramienta muy poderosa.

Algunas características con que deben contar los indicadores de acuerdo a Mondragón son: base teórica, especificidad, explicitud, disponibilidad para la comparabilidad, relevancia y oportunidad, no exclusividad de acción, claridad comprensiva, repetibilidad, solidez (válido, confiable, comparable y factible), sensibilidad -ante cambios-, costeo y economicidad.

Gutiérrez Espeleta realiza una revisión crítica de la evolución de los indicadores sociales y cuáles han sido los parámetros bajo los cuales se han desarrollado. México ha sido un país que ha retomado las definiciones y recomendaciones internacionales. Este autor refiere una definición de indicador "[...] es un instrumento construido a partir de un conjunto de estadísticas, que nos permite relatar una historia sobre fenómenos que no son evidentes ni medibles directamente" (Gutiérrez Espeleta, 2002:113).

Respecto de la sustentabilidad en México, un texto básico y clarificador es la publicación del Instituto Nacional de Estadística y Geografía (INEGI) del año 2000 titulado "Indicadores de Desarrollo Sustentable en México". En este compilado se relata la participación de México en la elaboración de indicadores del tipo de medición de fenómenos alineados a la sustentabilidad. Prácticamente se hace referencia a que el proceso no fue sencillo; varios fueron los obstáculos superados, así como varios los esfuerzos para incorporar las mejores prácticas vertidas desde la ONU para el desarrollo de indicadores sustentables.

López (2008:30) respecto del "marco conceptual, metodológico y normativo" comenta que es el "conjunto de bases conceptuales, guías metodológicas y normas y estándares nacionales e internacionales sobre medio ambiente y desarrollo sustentable puestas en práctica para la elaboración de estadísticas e indicadores y la integración de estos a los sistemas de información ambiental." Respecto de las estadísticas ambientales dice que "[...] describen el estado y las tendencias del ambiente, cubriendo los medios naturales (aire/clima, agua, tierras/suelos), la biota que se encuentra dentro de estos medios, así como los asentamientos humanos (ONU, 1985)" (López, 2008:31). Respecto del discurso internacional de consenso sobre desarrollo sustentable: "la construcción de indicadores de desarrollo sustentable, se ha basado en guías metodológicas en temas de interés nacional, regional y local, y también en temas que son de preocupación internacional" (López, 2008:43). Como resultado de lo anterior, se retoma del autor López el diagrama Indicadores sobre sustentabilidad ambiental urbana (véase Figura No. 1) que señala su propuesta de los ámbitos necesarios para la construcción de estos.

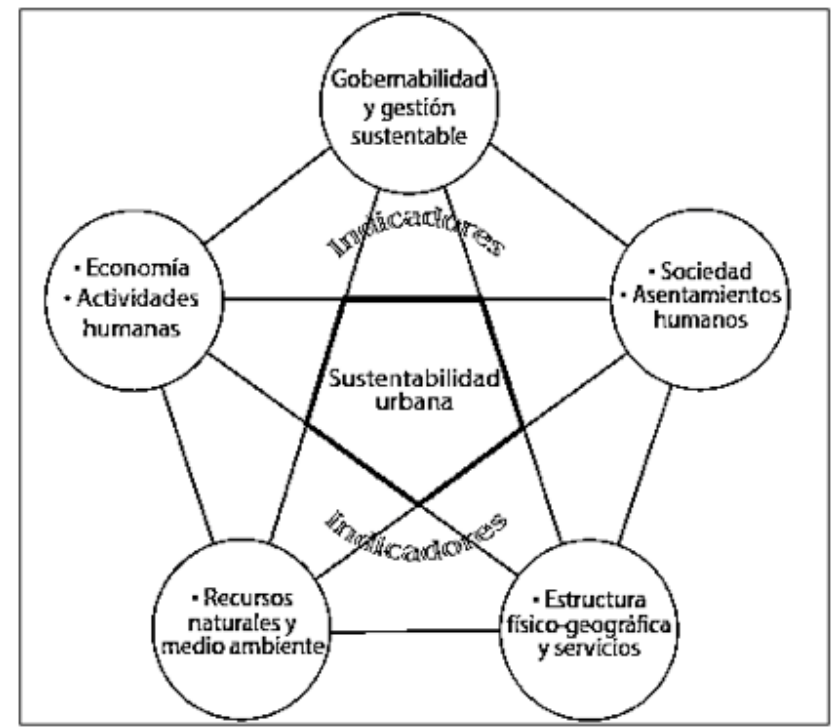

Figura 1. Indicadores sobre sustentabilidad ambiental urbana. Fuente: López Pérez, Carlos Roberto (2008:52).

Las ciudades demandan servicios, el crecimiento de éstas requiere de más insumos (recursos naturales, uso del ecosistema) para su mantenimiento. En la publicación de Indepth report: indicators for sustainable cities (2015) se plantean enfoques como el de metabolismo urbano, una identificación de elementos que son el motor de la ciudad y que en el mejor de los casos lo que se buscaría sería no sólo el buen funcionamiento de esta, sino un uso racional o por lo menos un equilibrio de los recursos naturales, que en este caso provee el ecosistema.

En la publicación Sustainable Urban Systems: articulating a long-term convergence research agenda (2018) se describen dos conceptos fundamentales que ayudarán a los investigadores a delimitar y estructurar sus propuestas de indicadores urbanos sustentables en pro de la construcción de ciudades sustentables: 
- Los sistemas urbanos son áreas geográficas con una alta concentración de actividad e interacciones humanas, integradas dentro de sistemas interdependientes multiescala, sociales, de ingeniería y naturales, que impactan el bienestar humano y planetario en escalas espaciales (locales a globales) y temporales.

- Los Sistemas Urbanos Sostenibles (SUS) son aquellos que están transformando sus estructuras y procesos con el objetivo de avanzar mensurablemente en el bienestar de las personas y el planeta.

\subsection{Transición hacia las energías limpias}

Gran parte de la historia de la evolución del desarrollo sustentable que investigadores como Víctor L. Urquidi documentaron en copiosa obra, se resume al intento internacional más actual por abatir las consecuencias de un modelo de crecimiento económico que sigue intentando cumplir con las promesas de la sustentabilidad (Agenda 2030). Este fue un compromiso global encabezado por la ONU, iniciado el 25 de septiembre de 2015 cuyo documento oficial "Transformar Nuestro Mundo: la Agenda 2030 para el Desarrollo Sostenible" ha sido adoptado por los 193 Estados Miembros de la ONU. Esta agenda revitaliza a los objetivos del milenio, siendo ahora 17 los Objetivos del Desarrollo Sostenible (ODS). En estricto, el relacionado con el tema de la energía asequible y no contaminante es el ODS número 7.

Son oportunas las preguntas ¿por qué las energías renovables en México? y ¿cómo es que éstas se justifican?, o bien ¿cuál es el marco jurídico que les da soporte? Las respuestas provienen del establecimiento de la Estrategia Nacional de Cambio Climático (ENCC) (DOF 03/06/2013) la cual es un reconocimiento directo al fenómeno global cambio climático. Esta estrategia nacional establece los lineamientos que en materia de combate al cambio climático deben diseñarse, implementarse, ejecutarse y evaluarse para que México pueda contribuir a la disminución respecto de sus emisiones de GEI, siendo uno de los lineamientos transitar a energías renovables. En el caso del gobierno mexicano, este amplía las opciones utilizando la clasificación de energías limpias (se incorpora a la energía nuclear y al gas natural), también para facilitar un manejo conveniente del discurso oficial.

Además de la ENCC y la reforma energética 2013/2014, se señalan otros sucesos importantes que han construido (de forma directa o indirectamente) el escenario actual de las energías limpias en México:

- Creación de la Comisión Nacional para el Uso Eficiente de la Energía (CONUEE) (28/11/2018).

- Ley general del cambio climático (06/06/2012).

- Creación del Instituto Nacional de Ecología y Cambio Climático (INECC) (06/06/2012).

- Inicia operaciones el Centro Mexicano en Innovación en Energía (CEMIE) Solar (14/02/2014).

- Ley de la industria eléctrica y Ley de energía geotérmica (11/08/2014).

- $\quad$ Acuerdo de Paris (12/12/2015).

- Ley de la transición energética (24/12/2015).

- Entra en funcionamiento el Mercado Eléctrico Mayorista (MEM) (23/03/2016).

El marco jurídico mexicano manifiesta que las energías limpias son parte de la solución al problema de los GEI, que coadyuvan con el desarrollo sustentable. Sin embargo, hay otras justificaciones alternativas que vale la pena considerar al momento de tratar de entender el proceso de transición hacia las energías limpias. Una de ellas es la teoría del cenit del petróleo, que señala que el recurso no renovable ya alcanzó su nivel máximo de producción y que más bien se encuentra en su fase de una constante y permanente caída. El proceso de transición energética de la mano de la reforma energética 2013/2014 ha sido un proceso complejo, lo sigue siendo ahora de cara al proceso de cambio de gobierno federal en el año 2018. Tema relacionado, debiera imperar la consideración de una matriz energética robusta, diversificada y sostenible, cuestión inclusive de seguridad energética.

Otra de las justificaciones de la conveniencia de transitar hacia las energías limpias recae en el hecho del aumento de la población urbana; las ciudades como "generadoras de oportunidades" concentrarán para 2050 -de acuerdo a escenarios de Hábitat III- al doble de la población hoy existente.

Retomando de Medina (2017:171): la demanda de energía se estima que crezca a razón de $1.8 \%$ anual hasta el año 2030 , lo que supone un crecimiento del $55 \%$ sobre las necesidades energéticas globales que se tienen en la actualidad; y, en el caso de las energías renovables, éstas incrementarán de $7 \%$ en 2012 al 20\% en 2020 (Roldán, 2012).

En competitividad global en materia de electricidad, México ocupa la posición 57; en cuanto al suministro de energía eléctrica ocupa el lugar 73; y en cuanto a obtención de electricidad ocupa el lugar 72 de 150 países.

De todas las justificaciones, ninguna pasa por cambiar los hábitos de consumo. Las energías renovables debieran suponer una oportunidad para un cambio en la forma en que hacemos uso de la energía eléctrica, sin embargo, éste es un tema pendiente, discutido con profundidad sólo en los ámbitos de la academia. Se entiende la complejidad de seguir promoviendo un modelo de crecimiento económico y de no poder dejar de consumir electricidad.

Para colocar en un marco de referencia el tema del monitoreo de la implementación y cobertura de paneles solares -a través del IICS- por estado de la República, es conveniente retomar de Medina (2017:176) el enfoque de eficiencia energética desde el contexto de las "buenas prácticas" y de la energía limpia, siendo tres los elementos considerados (véase Figura No. 2): tecnología eficiente, consumo de energía eléctrica y política verde.

El primer aspecto refiere tecnología eficiente, la adopción e implementación de la tecnología más actual, es decir, el máximo aprovechamiento de las energías limpias tiene que venir de la mano del desarrollo tecnológico y adopción de innovaciones. Se entiende por esto, tecnología que permita un mejor aprovechamiento de la energía. La fórmula también implica la necesidad de recurso humano capacitado.

El segundo aspecto consiste en contar con un sistema lo bastante consolidado de indicadores que nos permitan conocer del mercado, su consumo y la demanda.

El tercer aspecto se refiere a que la adopción, implementación y uso de las energías limpias sólo se puede ver favorecido en un esquema de incentivos, es decir, la transición debe fomentarse a través de política pública (de enfoque verde) clara y precisa. 


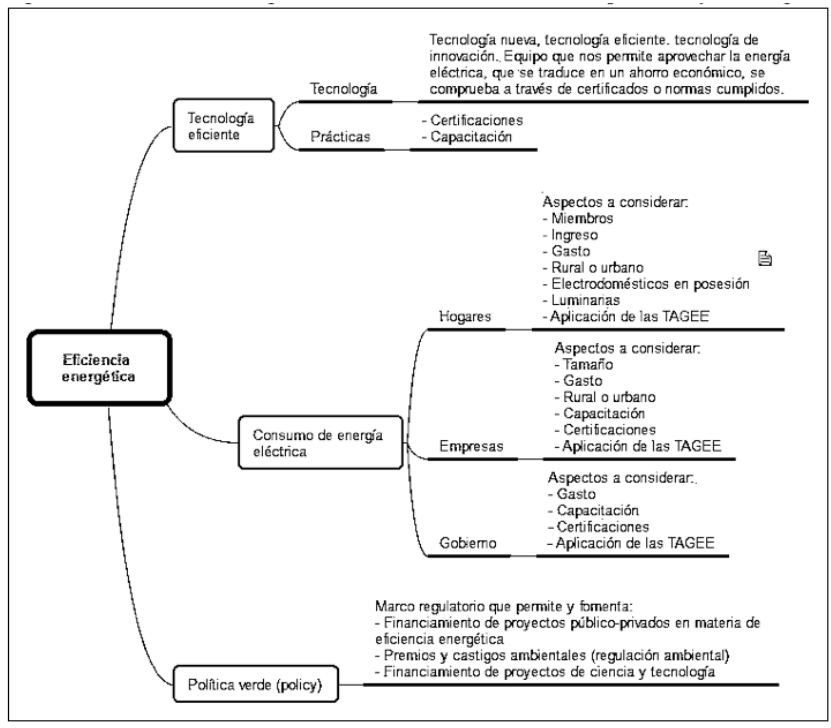

Figura 2. Eficiencia energética desde el contexto de las "buenas prácticas" y las energías limpias. Fuente: Medina (2017:176)

El propósito de este texto es concentrarse en los hogares mexicanos que se alimentan de energía eléctrica a través de paneles solares. Los datos que arroja la Encuesta Nacional de Gastos de los Hogares (ENGASTO) 2013 del INEGI, es que solamente 13 estados reportaban hogares con panel solar (véase la Tabla No. 1), siendo Sonora el Estado puntero en el fomento de paneles solares en los hogares, seguido por Durango, Chihuahua, Jalisco y Tamaulipas.

\subsection{Antecedentes de mecanismos fomentadores de paneles solares en México}

Para el caso de la industria del tipo pequeña y mediana empresas (PyMEs), uno de los organismos que ha atendido a este sector es el Fideicomiso para el Ahorro de la Energía Eléctrica (FIDE). En años recientes este organismo ha incorporado nuevos mecanismos de financiamiento que posibilitan la instalación de paneles solares (el FIDE identifica esta tecnología como ecotecnologías), tal es el caso del "Programa de Mejoramiento Integral Sustentable" con cobertura limitada a algunos estados de la República.

Bajo el mismo esquema de la eficiencia energética, la CONUEE atiende al sector de los gobiernos municipales, así como a la administración pública federal.

Como un beneficio de la Reforma Energética respecto de nuevos participantes en el MEM, para 2018 ya se encuentra disponible el servicio de instalación de paneles solares en los hogares con cargo al recibo telefónico (caso TELMEX), opción inicialmente (al 29 de febrero de 2018) disponible sólo para los estados de Ciudad de México, Querétaro, Morelos, Puebla, Hidalgo y Estado de México.

Un antecedente para el ámbito rural y con justificación relacionada con el tema de pobreza energética en México, es el que a continuación se retoma de Medina (2017): el 31 de mayo de 2017 la SENER y el FIDE publicaron la 1er. Convocatoria del Fondo de Servicio Universal Eléctrico (FSUE) cuyo objetivo es "beneficiar con energía fotovoltaica a 898 localidades rurales o urbanas marginadas de México que aún no cuentan con electrificación y a donde no es técnica ni económicamente factible extender la red de distribución de la CFE [...] mediante la modalidad de Sistemas Aislados [...]".

De acuerdo con la Secretaría de Energía (SENER) en el año 2014 la generación de energía solar en México fue de 135.5 GWh, siendo la capacidad instalada de 105 MW11, un registro muy bajo dentro del ámbito de las energías limpias. En 2014, de acuerdo con la Base de Indicadores de Eficiencia Energética (BIEE) de la CONUEE, el consumo de energía solar en el sector residencial fue de 1.26 TWh. De acuerdo con la ENGASTO del año 2013 del INEGI, se cuenta con el registro del número de hogares con energía eléctrica por panel solar (véase Tabla No. 1), sin embargo, se desconoce una variable que sería de utilidad, la generación de energía eléctrica por panel solar en hogar.

\section{Tabla 1. Hogares con energía eléctrica por panel solar en el año 2014.} Fuente: ENGASTO (INEGI, 2013)

\begin{tabular}{ccc}
\hline P & Entidad & $\begin{array}{l}\text { Hogares con } \\
\text { EE por panel solar } \\
(2014)\end{array}$ \\
\hline 1 & SON & 16,038 \\
2 & DGO & 6,914 \\
3 & CHIH & 5,954 \\
4 & JAL & 5,769 \\
5 & TAMPS & 5,017 \\
6 & ZAC & 3,130 \\
7 & NAY & 1,939 \\
8 & BCS & 1,496 \\
9 & HGO & 841 \\
10 & SLP & 524 \\
11 & CAM & 502 \\
12 & CAM & 496 \\
13 & GTO & 17 \\
\hline
\end{tabular}

\section{Desarrollo}

De acuerdo con los fundamentos explicados en el epígrafe anterior, proponemos desarrollar un indicador sustentable que lleve por nombre Indicador Innovación y Cobertura Solar (IICS).

De acuerdo con los tres ámbitos de la eficiencia energética descritos en la Figura No. 2 (Eficiencia energética desde el contexto de las "buenas prácticas" y las energías limpias), los indicadores a utilizar para el IICS (véase descripción en Tabla No. 2 - Las variables y los indicadores del IICS) comparten las siguientes características:

- Son observaciones estatales correspondientes al año 2013 y 2014.

Son en positivo (estado deseable).

Son valores relativos.

- En el caso de la información residencial (variable $\mathrm{X} 2$ : consumo de energía eléctrica), ésta corresponde al consumo o bien la demanda de energía eléctrica.

Son transversales.

- Cumple con los principios básicos de un indicador.

\subsection{Descripción de la hechura del indicador}

El IICS se compone de tres ámbitos, los mismos que ya fueron descritos de acuerdo con el enfoque de "Eficiencia energética desde el contexto de las 'buenas prácticas' y las 
energías limpias" (véase Figura No. 2). Los ámbitos se conforman de indicadores que cumplen con las características mínimas como disponibilidad y economía, es decir, las fuentes de donde han sido tomados son oficiales, INEGI (véase Tabla No. 2). Cada ámbito corresponde a una variable.

Tabla 2. Las variables y los indicadores del IICS

\begin{tabular}{|c|c|c|c|}
\hline Variable & Indicador & Fuente & Pesos \\
\hline \multirow{5}{*}{$\begin{array}{l}\text { X1: } \\
\text { Tecnología } \\
\text { eficiente } \\
\text { (peso 0.6) }\end{array}$} & $\begin{array}{l}\text { Hogares con EE por } \\
\text { panel solar }\end{array}$ & $\begin{array}{c}\text { ENGASTO } \\
2013\end{array}$ & 0.6 \\
\hline & $\begin{array}{l}\text { PyMEs con } \\
\text { Certificación en } \\
\text { Medio }\end{array}$ & $\begin{array}{l}\text { ENAPR } \\
\text { OCE }\end{array}$ & 0.3 \\
\hline & $\begin{array}{l}\text { Ambiente } \\
\text { PyMEs que } \\
\text { ofrecieron capaci_- } \\
\text { tación a su } \\
\text { personal }\end{array}$ & $\begin{array}{l}2013- \\
2014\end{array}$ & 0.1 \\
\hline & $\begin{array}{l}\text { Hogares con EE por } \\
\text { servicio público }\end{array}$ & & 0.5 \\
\hline & $\begin{array}{l}\text { Hogares con EE por } \\
\text { planta particular }\end{array}$ & & 0.05 \\
\hline \multirow{9}{*}{$\begin{array}{l}\text { X2: Consumo } \\
\text { de energía } \\
\text { eléctrica } \\
\text { (peso 0.2) }\end{array}$} & Hogares con & & 0.05 \\
\hline & $\begin{array}{l}\text { Hogares con horno de } \\
\text { microondas }\end{array}$ & $\begin{array}{c}\text { ENGASTO } \\
2013\end{array}$ & 0.05 \\
\hline & $\begin{array}{l}\text { Hogares con } \\
\text { refrigerador }\end{array}$ & & 0.05 \\
\hline & $\begin{array}{l}\text { Hogares con lava } \\
\text { dora }\end{array}$ & & 0.05 \\
\hline & Hogares con radio & & 0.05 \\
\hline & Hogares con TV & & 0.05 \\
\hline & $\begin{array}{l}\text { Hogares con } \\
\text { conexión a internet }\end{array}$ & & 0.05 \\
\hline & $\begin{array}{l}\text { Tamaño medio del } \\
\text { hogar }\end{array}$ & & 0.05 \\
\hline & $\begin{array}{l}\% \text { de gasto promedio } \\
\text { hogar en servicios }\end{array}$ & & 0.05 \\
\hline \multirow{5}{*}{$\begin{array}{l}\text { X3: Política } \\
\text { verde (peso } \\
0.2 \text { ) }\end{array}$} & $\begin{array}{l}\text { UE encuestadas que } \\
\text { aplicaron }\end{array}$ & & 0.5 \\
\hline & innovación en al & CE 2014 & \\
\hline & menos un año de & & \\
\hline & $\begin{array}{l}2011 \text { a } 2013 \text { (en el } \\
\text { sector } 22)\end{array}$ & & \\
\hline & $\begin{array}{l}\text { UE encuestadas con } \\
\text { gasto o inversión en } \\
\text { investigación y } \\
\text { desarrollo }\end{array}$ & & 0.5 \\
\hline
\end{tabular}

El proceso de normalización para los indicadores consistió en tomar el valor máximo como "el mejor". Respecto de la asignación de "los pesos" a indicadores, se asignó un alto peso a aquellos de contenido informativo relacionado con los paneles solares, posterior en prioridad, recurso humano capacitado; luego, unidades económicas que hayan implementado algún proceso de innovación.

Posteriormente "los pesos" para las variables X1, X2 y X3 se asignaron como 0.6 para tecnología eficiente (X1), 0.2 para consumo de energía eléctrica (X2), y 0.2 para política verde (X3). Los pesos de las tres variables suman 1.0

Consideramos la adopción, implementación y utilización en este caso paneles solares- como acciones conjuntas/secuenciales básicas para iniciar un proceso de transición hacia las energías limpias, inclusive aquellas distintas a las tecnologías solares.

Para iniciar un proceso de adopción de nuevas tecnologías se requiere que el usuario y/o consumidor de energía eléctrica experimente y compruebe que las energías renovables -a través de tecnologías como el kit de paneles solares- son una realidad en su hogar, además que son convenientes (para el usuario final económicamente hablando respecto al costo beneficio), seguras, asequibles y prácticas. Lo anterior implica que el gobierno incentive la adopción, implementación y uso de las tecnologías renovables -en éste caso paneles solares en los hogares-, lo cual justifica la consideración de una política verde. Finalmente, para la implementación de tecnologías como los panes solares es importante tener en conocimiento la demanda de energía eléctrica de los hogares.

\subsection{Resultados del ejercicio}

Del top ten de estados arrojado por el IICS para el año 2014 (véase la Tabla No. 3), se observa que ocho de ellos son estados que para el año mencionado reportaron la mayor cantidad de hogares con energía eléctrica por panel solar: Sonora, Durango, Chihuahua, Tamaulipas, Zacatecas, Nayarit, Baja California Sur y Campeche. Es decir, se observa una coincidencia de un alto valor del IICS versus los estados que han ejecutado una puntual política de fomento de hogares con panel solar.

Tabla No. 3 - Ranking del IICS para el año 2014

\begin{tabular}{|c|c|c|c|c|c|}
\hline $\mathrm{P}$ & Entidad & $\begin{array}{c}\text { Ranking } \\
\text { IICS }\end{array}$ & $\mathrm{P}$ & Entida d & $\begin{array}{c}\text { Ranking } \\
\text { IICS }\end{array}$ \\
\hline 1 & DGO & 64.50 & 17 & $\mathrm{COAH}$ & 31.94 \\
\hline 2 & SON & 64.40 & 18 & YUC & 31.93 \\
\hline 3 & $\mathrm{ZAC}$ & 50.71 & 19 & $\mathrm{COL}$ & 31.84 \\
\hline 4 & CHIS & 49.04 & 20 & GRO & 31.80 \\
\hline 5 & MOR & 43.41 & 21 & QROO & 31.64 \\
\hline 6 & NAY & 43.08 & 22 & OAX & 31.58 \\
\hline 7 & BCS & 41.74 & 23 & QRO & 31.15 \\
\hline 8 & $\mathrm{CHIH}$ & 41.69 & 24 & CDMX & 30.76 \\
\hline 9 & TMAPS & 37.45 & 25 & GTO & 30.37 \\
\hline 10 & CAM & 37.36 & 26 & $\mathrm{BC}$ & 30.32 \\
\hline 11 & $\mathrm{HGO}$ & 36.69 & 27 & SLP & 30.06 \\
\hline 12 & JAL & 35.97 & 28 & AGS & 30.04 \\
\hline 13 & TAB & 35.73 & 29 & NL & 29.98 \\
\hline 14 & VER & 35.29 & 30 & MEX & 29.53 \\
\hline 15 & $\mathrm{MICH}$ & 34.77 & 31 & TLAX & 28.12 \\
\hline 16 & PUE & 32.20 & 32 & SIN & 27.61 \\
\hline
\end{tabular}

Del top ten arrojado por el IICS se observa que los estados de Chiapas y Morelos, a pesar de no tener registro de hogares con panel solar en el año 2014, el puntaje en otros rubros del IICS fue lo suficientemente significativo como para permitir que trascendieran en la lista del top ten del IICS.

Los datos arrojados para 2014 por el IICS lleva a realizar diversas conjeturas, una de ellas es que los estados con mayor aportación al Producto Interno Bruto (PIB), como sería el caso de la Ciudad de México y Estado de México, no 
necesariamente son los que más contribuyen al rubro de las energías renovables, al menos no desde el rubro de fomentar la energía solar en los hogares, ¿a qué se debe esta situación?

\section{Conclusiones}

En la publicación Urbanización y desarrollo, futuros emergentes de la ONU de 2016, se destaca aquel principio de "la ciudad es prosperidad", ésta posee un "poder transformador de cambiar vidas", en la que se establece la idea de que a mayor urbanización menor pobreza.

Si bien la idea anterior acompaña al discurso oficialista del desarrollo urbano sustentable, existe la posibilidad de que dicha relación sea no sólo cierta, sino que sea posible contribuir a que se cumpla; aquí los indicadores urbanos de sustentabilidad tienen una participación real de incidir en los procesos conformadores de mejor ciudad (a través de por ejemplo, conceptos como ciudad sostenible, ciudad sustentable, ciudad inteligente, ciudad resiliente), pero ¿en qué grado y a qué ritmo?, eso dependería del planteamiento básico de ¿qué modelo de ciudad sustentable queremos?

Respecto del tema del crecimiento de las ciudades, son varios los planteamientos que surgen. Uno de ellos es que a sabiendas de la importancia actual y futura de las ciudades, sobre todo por la alta concentración de personas y los recursos en términos de servicios urbanos que éstas demandarán, lleva a plantearse ¿cómo es que va a suceder esto? Nuevamente los indicadores urbanos sustentables, en este sentido, pueden contribuir.

El ejercicio exploratorio de realizar un indicador como el IICS, permite reflexionar sobre varios aspectos: el primero de ellos el referente a la información, es decir, si bien se cuenta con información disponible que permite aproximar algunos resultados respecto del "estado" actual de las energías renovables en México, en específico la solar, la realidad es que se desearía contar con información más desagregada, más específica respecto de cómo los usuarios de las nuevas tecnologías solares en los hogares hacen uso de estos nuevos implementos y cómo se están beneficiando. Respecto de los cursos de capacitación y certificaciones de las PyMEs, sería muy oportuno conocer cuáles de estas corresponden a temas relacionados en estricto con las energías renovables, y en este caso, con energía solar; esto daría a nuestro indicador (y a otros similares) mayor certidumbre.

El segundo aspecto de reflexión tiene que ver con el "estado" actual de las propias energías renovables en nuestro país, respecto de su penetración en el mercado de los usuarios potenciales. Se podría justificar que siendo el periodo analizado el año 2014 el mismo de la reforma energética, se hayan encontrado datos reveladores de una baja implementación de paneles solares. Si bien se mencionó que para el año 2014 trece eran los estados que reportaban hogares con energía eléctrica por panel solar (heeps), siendo Sonora el estado puntero. La realidad es que en cifras relativas, retomando el caso de Sonora con sus 16,038 heeps, esta cifra sólo representa el $1.82 \%$ del total de los hogares de ese estado.

El realizar el comentario de que la Reforma Energética y la consecuente andanada de procesos ejecutados "a raíz de" no es cosa menor. El año 2014 deberá ser considerado por los especialistas como un año base respecto de las informaciones de las energías renovables, incidiendo por consecuencia en los comparativos entre indicadores propios de la materia de antes de 2014 y después de ese año. Quizás los indicadores más recurridos del sector eléctrico, generación bruta y capacidad instalada, necesariamente se verán afectados por una diversificación de sus componentes y fuentes de información. Es posterior al año 2014 que al adecuarse metodologías de almacenamiento y proceso de información por parte de los organismos encargados, que posiblemente conoceremos información más puntual y desagregada considerando el despliegue -en información- del amplio abanico de las energías limpias en México.

\section{English Summary}

\section{Indicator of innovation and solar coverage}

\begin{abstract}
This text recognizes the importance of sustainable urban indicators, as well as the broad and very extensive context, which over the years has had sustainable development, which along with climate change are the requesting and at the same time promoters of indicators that contribute to the improvement of the quality of life of the people who live in the cities. Energy is fundamental for the development of societies, the objective of sustainable development number seven is the official interest of the UN in this matter, the framework is the 2030 Agenda. Of the set of renewable energies, it is the solar option in conjunction with the solar panel technological addition, the option that has the greatest potential for implementation in the domestic sphere. Then, according to the described scenario, it has been the task to develop an exploratory indicator to know the coverage of solar energy in Mexican households, using as a first input households with electric energy by solar panel. We return to the proposed approach "energy efficiency from the context of 'good practices' and clean energy." Under the aforementioned approach we group the relative data, normalize it and weigh it. We do a national review for 2014 using data from INEGI. You get a ranking then, Durango and Sonora are the first on the list. Some reflections are provided regarding the exercise and the existing opportunity areas.
\end{abstract}

\section{Keywords:}

Solar energy, solar panel, homes with solar panel, urban indicator.

\section{Referencias}

Altamirano, Juan (1992). Algunos "paradigmas" en la operacionalización de los conceptos en Paul Lazarsfeld. Temas Sociales [online]. No. 16, pp. 139-150.

Environmental Research \& Education (2018). Sustainable Urban Systems: articulating a long-term convergence research agenda.

Gutiérrez Garza, Esthela (2012). El desarrollo sustentable: una gran convocatoria internacional. En Indicadores de sustentabilidad en el estado de Nuevo León. México. Siglo Veintiuno Editores. pp. 13-16.

Gutiérrez-Espeleta, Édgar E (2002), Capítulo II - Indicadores sociales: una breve interpretación de su estado de desarrollo, En Sojo, Carlos, 
Desarrollo Social en América Latina: temas y desafíos para las políticas públicas. FLACSO. pp. 105-149.

Home, Rollo (2014), IX Modelling resilience: The importance of geospatial data. En Cole, Jennifer (2014) Measuring the resilience of cities, the role of big data. Londres. Science \& Technology. pp. 73-81

INEGI (2000). Indicadores de desarrollo sustentable en México. México.

Lazarsfeld, Paul. (1973), Capítulo I - De los conceptos a los índices empíricos. En Boudon, Raymond y Lazarsfeld Paul (1973), Metodología de las ciencias sociales, Volumen I. España. Editorial Laia. pp. 35-46.

López Pérez, Carlos Roberto (2008) Experiencia del INEGI en la elaboración de indicadores ambientales y de desarrollo sustentable, En López Blanco, J. y Rodríguez Gamiño, M., Desarrollo de indicadores ambientales y de sustentabilidad en México. México. UNAM Instituto de Geografía. pp. 27-55.
Mondragón Pérez, Angélica Rocío (2002). ¿Qué son los indicadores?, En Revista de información y análisis, No. 19.

ONU Hábitat (2016). Urbanización y desarrollo, futuros emergentes.

Roldán Viloria, José et al. (2012). Energías renovables, lo que hay que saber. España. Ediciones paraninfo.

Science for environment policy (2015). In-depth report: indicators for sustainable cities.

SENER (2017). Programa Especial de la Transición Energética $2017-$ 2018.

SENER. PRODESEN 2016-2030.

DOF (03/06/2013). Estrategia Nacional de Cambio Climático.

Medina Josefa, Erwin (julio-diciembre 2017), Hogares mexicanos con panel solar, Revista legislativa de estudios sociales y de opinión pública, Vol. 10, Núm. 20, pp. 169-196 